\title{
Comparison between multiparametric MRI with and without post - contrast sequences for clinically significant prostate cancer detection
}

Thais Caldara Mussi ${ }^{1}$,Tatiana Martins ${ }^{1,2}$, George Caldas Dantas ${ }^{1}$, Rodrigo Gobbo Garcia ${ }^{3}$, Renee Zon Filippi ${ }^{4}$, Gustavo Caserta Lemos ${ }^{5}$, Ronaldo Hueb Baroni ${ }^{1}$

${ }^{1}$ Departamento de Radiologia e Diagnóstico por Imagem, Hospital Israelita Albert Einstein, SP, Brasil;

${ }^{2}$ Ecoar Medicina Diagnóstica, Lourdes, Belo Horizonte, MG, Brasil; ${ }^{3}$ Departamento de Intervenção Guiada por Imagens, Hospital Israelita Albert Einstein, SP, Brasil; ${ }^{4}$ Departamento de Patologia, Hospital Israelita Albert Einstein, SP, Brasil; ${ }^{5}$ Departamento de Urologia, Hospital Israelita Albert Einstein, SP, Brasil

\section{ABSTRACT}

Background: Dynamic-contrast enhanced (DCE) sequence is used to increase detection of small lesions, based on increased vascularization. However, literature is controversy about the real incremental value of DCE in detection of clinically significant (CS) prostate cancer $(\mathrm{PCa})$, since absence of enhancement does not exclude cancer, and enhancement alone is not definitive for tumor. Purpose: To test the hypothesis that DCE images do not increase CS PCa detection on MRI prior to biopsy, comparing exams without and with contrast sequences. Material and Materials and Methods: All men who come to our institution to perform MRI on a 3T scanner without a prior diagnosis of CS PCa were invited to participate in this study. Reference standard was transrectal prostate US with systematic biopsy and MRI/US fusion biopsy of suspicious areas. Radiologists read the MRI images prospectively and independently (first only sequences without contrast, and subsequently the entire exam) and graded them on 5-points scale of cancer suspicion.

Results: 102 patients were included. Overall detection on biopsy showed CS cancer in 43 patients (42.2\%), clinically non-significant cancer in $11(10.8 \%)$ and negative results in 48 patients (47\%). Positivities for CS PCa ranged from $8.9 \%$ to $9.8 \%$ for low suspicion and $75.0 \%$ to $88.9 \%$ for very high suspicion. There was no statistical difference regarding detection of CS PCa (no statistical difference was found when compared accuracies, sensitivities, specificities, PPV and NPV in both types of exams). Inter-reader agreement was 0.59 .

Conclusion: Exams with and without contrast-enhanced sequences were similar for detection of CS PCa on MRI.

\section{ARTICLE INFO}

\section{Keywords:}

Magnetic Resonance Imaging;

Prostatic Neoplasms; Men

Int Braz J Urol. 2018; 44: 1129-38

Submitted for publication:

February 16, 2018

Accepted after revision:

June 17, 2018

Published as Ahead of Print:

September 20, 2018

\section{INTRODUCTION}

Prostate cancer (PCa) is a major global health problem, as the most common cancer in men, aside from skin cancer, and the second - le- ading cause of cancer death in the United States (1). Approximately 30\% of men older than 50 years of age have pathologic evidence of PCa; however, only $3 \%$ will die from their disease $(2,3)$. 
The diagnosis of PCa increased in the mid 1980's when prostate - specific antigen (PSA) became a screening biomarker. However, PSA screening is a cause of over diagnosis and consequent overtreatment of patients with indolent disease (2). Therefore, the recommendation to use PSA for PCa screening remains controversial (4).

Efforts have been made to better define the clinical behavior of prostate tumors, which can range from indolent and clinically insignificant (CI) tumors to aggressive and metastatic cancer $(5,6)$.

Benefits of multiparametric magnetic resonance imaging (mpMRI) in patients with clinical suspicion of PCa are already established (7). MpMRI has the ability to improve detection of clinically significant (CS) PCa and decrease the detection of CI tumors prior to biopsy (7-9). Some studies already demonstrated that mpMRI is the best predictor for CS PCa detection $(10,11)$. Additionally, mpMRI used along with PSA has been shown to increase negative predictive values to rule out $\mathrm{PCa}$, making it an excellent test to avoid unnecessary biopsies in biopsy - naïve patients and men with prior negative biopsies (12-14).

A routine mpMRI should include T1 weighted (T1W), T2 - weighted (T2W), diffusion - weighted image (DWI), and dynamic contrast enhanced (DCE) sequences, as recommended by major international guidelines (15). T1W images are used to detect hemorrhages within the prostate and seminal vesicles. T2W images are mostly used to evaluate prostatic anatomy, detect morphological abnormalities, and evaluate extraprostatic extension and seminal vesicle invasion in cases of advanced tumors. DWI is helpful to differentiate CS PCa from benign lesions and predict cancer aggressiveness. It should be used in conjunction with the other sequences. Finally, DCE is used to increase detection of small lesions (13), based on increased vascularization of these lesions. However, the real incremental value of DCE in detection of CS PCa is controversial, since absence of enhancement does not exclude cancer, and enhancement alone is not definitive for tumor (16).

Regardless of its advantages and increased usefulness, mpMRI is expensive and time consuming Gadolinium introduces risk of allergic reac- tion, potential development of nephrogenic systemic fibrosis and deposition in brain tissue (11, 17-19). However, the clinical effects of deposition of this agent contrast in the brain are not know until nowadays.

The objective of our study is to test the hypothesis that contrast - enhanced images do not increase the detection of CS PCa on mpMRI prior to biopsy, comparing exams with and without contrast in the same patient population.

\section{MATERIALS AND METHODS}

\section{Study design}

From June 2015 until February 2016, all male patients who came to our institution to perform prostatic mpMRI without a prior diagnosis of CS PCa were invited to participate in this prospective, institutional review board approved study (CAAE number 40942915.7.0000.0071). All male patients included in this study signed informed consent.

Exclusion criteria were: prostate biopsy not performed or performed in another institution, incomplete mpMRI protocol, biopsy performed more than six months after mpMRI, and an exam that was not evaluated by the two radiologists of this study.

A total of 447 patients signed the informed consent to enter the study over a nine month period, and 345 were excluded for the following reasons: prostate biopsy not performed or performed in another institution ( $\mathrm{n}=339$ ), incomplete mpMRI protocol $(n=2)$, biopsy performed more than six months after mpMRI $(\mathrm{n}=1)$, and exams not read not by the two study radiologists $(n=3)$.

\section{Imaging}

All patients underwent mpMRI on a 3 Tesla scanner: Magnetom Prisma (Siemens Medical Solutions, Erlangen, Germany) or Discovery MR 750W (GE Healthcare, Little Chalfont, United Kingdom) with a phased array coil and without an endorectal coil. A routine protocol including triplanar T2W imaging, DWI (b - values $=50,400$, 800 and 1500) and DCE sequences were performed covering the prostate and seminal vesicles. Fifteen post - contrast sequences were acquired with 
a temporal resolution of 13 seconds each. Extracellular gadolinium - based contrast media (Magnevist, Bayer, Leverkusen, Germany) was injected at a dose of $0.2 \mathrm{cc} / \mathrm{Kg}$ and a rate of $2 \mathrm{cc} / \mathrm{sec}$.

\section{Biopsy protocol}

As reference standard, transrectal prostate ultrasound (US) systematic biopsy (14 - cores, 12 from peripheral zone and two from transition zone) and mpMRI / US fusion with additional samples of suspicious areas was adopted. US - guided biopsies were performed using either an Aplio 500 with Smart Fusion (Toshiba Medical System Corporation, Minato, Tokyo, Japan) or a LOGIC E9 with imaging fusion software (GE Healthcare, Little Chalfont, United Kingdom). One out seven radiologists with experience in prostate biopsy with imaging fusion mpMRI / US (minimum of 3 year of experience) performed the prostate biopsy, aware of mpMRI findings.

TOne out four of the pathologists from the hospital performed the histopathologic analysis, with at least 15 years of experience in uropathology. Histological findings were classified for each prostatic region as negative, positive CI tumor (Gleason $3+3$ ), or positive CS tumor (Gleason $\geq 3$ + 4) (20).

\section{Data analysis}

Two fellowship trained radiologists (with 6 and 15 years of experience in prostate mpMRI) read images prospectively and independently (blinded to each other): first they filled in a form classifying the prostate mpMRI in suspicion levels for PCa reading only sequences without contrast. Subsequently, they filled in another form reclassifying the suspicion levels for PCa reading the entire exam including the post - contrast enhancement sequences. Both radiologists were aware of the patient's clinical data. Analysis was performed into eight prostatic regions (apex, mid and base of peripheral zone; transition zone, right and left), and graded on 5 - point scale of cancer suspicion (1: CS PCa is very unlikely; 2 : CS is unlikely; 3 : presence of CS PCa is equivocal; 4: CS PCa is likely; and 5: CS PCa is very likely). A final consensus analysis was performed to make the final report, which was used to guide the suspicious areas on biopsies. The imaging-pathologic correlation was performed by one of the authors after all the MRI readings were finished (18).

\section{Statistical methods}

We performed a histogram analysis to verify the distribution. Because numeric variables were not normally distributed, they were described with median and interquartile range (IQR).

To verify the association between mpMRI categories (1-5) and biopsy results we used generalized estimating equations (21), with permutable correlation structures, using the software $\mathrm{R}$ 3.1.3 (R Core Team, 2015). Sensitivity, specificity, positive predictive value (PPV), negative predictive value (NPV) and accuracy for both readers were calculated using biopsy as reference standard. The level for statistical significance was set at 5\%.

Inter - reader agreement was calculated using Cohen's Kappa coefficient of agreement within ordinal weights, and it was defined as: excellent ( $\mathrm{k}$ $\geq 0.81)$, good $(\mathrm{k}=0.61-0.80)$, moderate $(\mathrm{k}=0.41$ $0.60)$, fair $(\mathrm{k}=0.21-0.40)$, and poor $(\mathrm{k} \leq 0.20)$.

\section{RESULTS}

The final cohort was comprised of 102 patients with a median age of 62.1 years old (range 35.1 - 82.1). Median time between mpMRI and biopsy was 15 days (IQR 14; 16); median PSA level was $4.36 \mathrm{ng} / \mathrm{mL}$ (IQR 3.19; 5.83); median number of fragments in the prostate biopsy was 19 (IQR 17; 21); and median number of fragments for each suspicious lesion was 4 (IQR 3; 5). Twenty - five patients (24\%) were submitted to prior biopsy, and of those, 19 (76\%) had negative results. The remaining 6 patients (24\%) were on active surveillance for a CI tumor (up to two fragments of Gleason $3+3$ on previous biopsy). Previous prostate biopsies were performed with a median time of 21 months (range 2 180 ) prior the mpMRI and those patients had no post - biopsy hemorrhage in the prostate gland during exam analysis.

Overall biopsy results showed CS cancer in 43 (42.2\%), CI cancer in 11 (10.8\%), and negative result for cancer in 48 (47\%) patients. Of the 
25 patients who had prior biopsies with negative results or CI tumors, 10 (40\%) had new diagnoses of CS tumors and one (4\%) maintained CI tumor diagnosis.
Each radiologist evaluated a total of 816 prostatic regions in each phase of the study (eight prostatic regions in 102 patients). Table-1 provides the mpMRI readings on the eight prostatic regions

Table 1 - Positivity results regarding the suspicion level on mpMRI in a sextant pattern.

\begin{tabular}{|c|c|c|c|c|c|c|c|c|c|c|c|c|}
\hline \multirow{3}{*}{ Radiologist } & \multirow{3}{*}{$\begin{array}{c}\text { MRI } \\
\text { category }\end{array}$} & \multirow{3}{*}{ Contrast } & \multirow{3}{*}{$\begin{array}{c}\text { Global } \\
\text { N }\end{array}$} & \multicolumn{9}{|c|}{ Biopsy } \\
\hline & & & & \multicolumn{3}{|c|}{ Negative } & \multicolumn{3}{|c|}{$\begin{array}{l}\text { Positive clinically non- } \\
\text { significant }\end{array}$} & \multicolumn{3}{|c|}{ Positive clinically significant } \\
\hline & & & & $\mathrm{n}$ & $\mathrm{p}(95 \% \mathrm{Cl})$ & $p$-value & $\mathrm{n}$ & $\mathrm{p}(95 \% \mathrm{Cl})$ & $\mathrm{p}$-value & $\mathrm{n}$ & $\mathrm{p}(95 \% \mathrm{Cl})$ & $p$-value \\
\hline \multirow{10}{*}{1} & 1 & With & 10 & 10 & 100.0 & - & 0 & 0.0 & - & 0 & 0.0 & - \\
\hline & & Without & 10 & 10 & 100.0 & & 0 & 0.0 & & 0 & 0.0 & \\
\hline & 2 & With & 504 & 435 & $86.3(83.3-89.3)$ & 0.969 & 21 & $4.2(2.4-5.9)$ & 0.868 & 48 & $9.5(7.0-12.1)$ & 0.876 \\
\hline & & Without & 530 & 457 & $86.2(83.3-89.2)$ & & 21 & $4.0(2.3-5.6)$ & & 52 & $9.8(7.3-12.3)$ & \\
\hline & 3 & With & 247 & 202 & $81.8(77.0-86.6)$ & 0.813 & 9 & $3.6(1.3-6.0)$ & 0.877 & 36 & $14.6(10.2-19.0)$ & 0.731 \\
\hline & & Without & 230 & 190 & $82.6(77.7-87.5)$ & & 9 & $3.9(1.4-6.4)$ & & 31 & $13.5(9.1-17.9)$ & \\
\hline & 4 & With & 36 & 20 & $55.6(39.3-71.8)$ & 0.187 & 2 & $5.6(0.0-13.0)$ & 0.984 & 14 & $38.9(23.0-54.8)$ & 0.185 \\
\hline & & Without & 28 & 11 & $38.9(20.9-56.9)$ & & 2 & $5.7(0.0-13.6)$ & & 15 & $55.8(37.1-74.5)$ & \\
\hline & 5 & With & 19 & 3 & $15.8(0.0-32.2)$ & 0.679 & 0 & 0.0 & - & 16 & $84.2(67.8-100.0)$ & 0.679 \\
\hline & & Without & 18 & 2 & $11.1(0.0-25.6)$ & & 0 & 0.0 & & 16 & $88.9(74.4-100.0)$ & \\
\hline \multirow{10}{*}{2} & 1 & With & 9 & 9 & 100.0 & - & 0 & 0,0 & - & 0 & 0.0 & - \\
\hline & & Without & 12 & 9 & 75.0 & & 0 & 0,0 & & 3 & 25.0 & \\
\hline & 2 & With & 482 & 418 & $86.7(83.7-89.8)$ & 0.819 & 19 & $3.9(2.2-5.7)$ & 0.984 & 45 & $9.3(6.7-11.9)$ & 0.799 \\
\hline & & Without & 485 & 423 & $87.2(84.2-90.2)$ & & 19 & $3.9(2.2-5.6)$ & & 43 & $8.9(6.3-11.4)$ & \\
\hline & 3 & With & 268 & 212 & $79.1(74.2-84.0)$ & 0.851 & 10 & $3.7(1.5-6.0)$ & 0.924 & 46 & $17.2(12.6-21.7)$ & 0.801 \\
\hline & & Without & 257 & 205 & $79.8(74.9-84.7)$ & & 10 & $3.9(1.5-6.3)$ & & 42 & $16.3(11.8-20.9)$ & \\
\hline & 4 & With & 38 & 27 & $71.1(56.6-85.5)$ & 0.673 & 3 & $7.9(0.0-16.5)$ & 0.899 & 8 & $21.1(8.1-34.0)$ & 0.590 \\
\hline & & Without & 42 & 28 & $66.7(52.4-80.9)$ & & 3 & $7.1(0.0-14.9)$ & & 11 & $26.2(12.9-39.5)$ & \\
\hline & 5 & With & 19 & 4 & $21.1(2.7-39.4)$ & 0.770 & 0 & 0.0 & - & 15 & $78.9(60.6-97.3)$ & 0.770 \\
\hline & & Without & 20 & 5 & $25.0(6.0-44.0)$ & & 0 & 0.0 & & 15 & $75.0(56.0-94.0)$ & \\
\hline
\end{tabular}

$\mathbf{M p}=$ multiparametric; $\mathbf{M R I}=$ magnetic resonance imaging; $\mathbf{N}=$ number of prostate regions; $\mathbf{9 5} \% \mathbf{C l}=\mathbf{9 5 \%}$ confidence intervals; $\mathrm{P}$-value to compare exams without and with contrast. 
that had no statistical difference regarding detection of CS PCa in exams with and without contrast for both readers. Positive CS PCa ranged from $8.9 \%$ to $9.8 \%$ for low suspicion (category 2) and $75.0 \%$ to $88.9 \%$ for very high suspicion (category 5) on mpMRI categories (Figure-1). The odds of having CS PCa on mpMRI was 2.75 (reader 1) and 2.4 (reader 2). In corroborating these findings, no as positive on mpMRI studies with and without contrast (Table-3).

Post - contrast sequences changed the overall mpMRI analysis in 11 cases for reader 1 (increasing the category in 10 cases) and in 7 cases for reader 2 (increasing the category in two cases). For reader 1, the post - contrast sequences correlated with biopsy results (positive enhancement in

Figure 1 - Seventy three years old man with PSA level of $3.4 \mathrm{ng} / \mathrm{mL}$ and normal DRE. MpMRI shows a 7 mm nodule in the left apical peripheral zone (T2-weighted imaging on A), with marked restricted diffusion seen on ADC map (B) and on b-value of 1500 (C). The lesion was categorized as very high suspicion for CS PCa (category 5) for both readers in both reading sessions despite the small size and DCE sequence. Early enhancement of the lesion is seen on DCE (D). Biopsy confirmed Gleason 4+4.

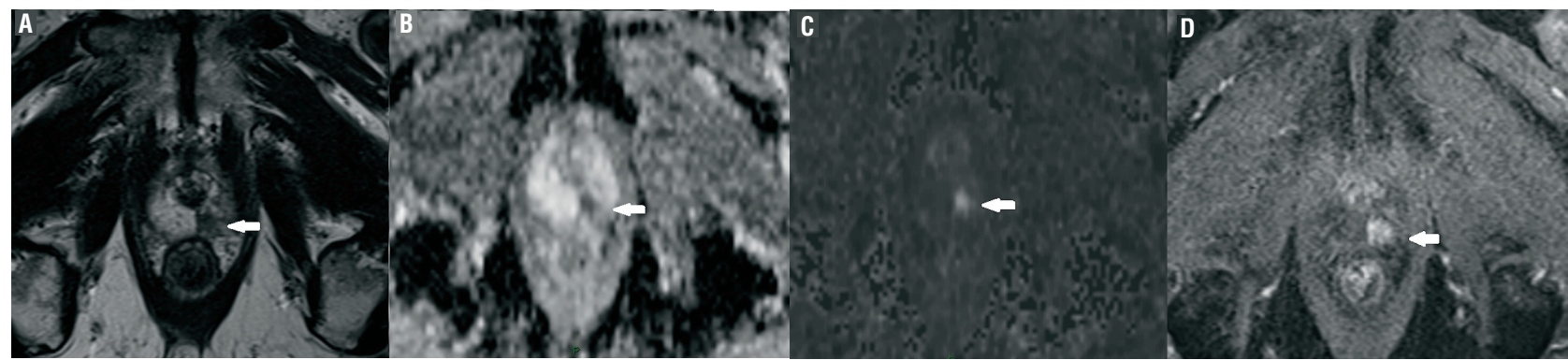

statistical difference was found when we compared accuracy, sensitivity, specificity, PPV, and NPV in both sets of exams (Table-2). Accuracy was slightly better in exams without contrast for both readers, but without statistical significance.

The best sensitivity and specificity values were obtained when including category 3 (equivocal)
CS tumors or negative enhancement in negative results / CI tumors) in 5 cases (45\%) and resulted in misclassification in 6 (55\%). For reader 2, the sequences correlated with biopsy results in two cases (29\%) and resulted in misclassification in five (71\%). Post - contrast sequences identified four regions with CS tumors more than

Table 2 - Diagnostic measurements.

\begin{tabular}{|c|c|c|c|c|c|c|c|c|c|c|c|}
\hline Radiologist & Contrast & Accuracy & P-value & Sensitivity & P-value & Specificity & P-value & PPV & P-value & NPV & P-value \\
\hline \multirow{2}{*}{1} & With & $\begin{array}{c}65.2 \\
(61.9-68.5)\end{array}$ & 0.346 & $\begin{array}{c}57.9 \\
(48.8-67.0)\end{array}$ & 0.594 & $\begin{array}{c}66.4 \\
(62.9-69.9)\end{array}$ & 0.208 & $\begin{array}{c}21.9 \\
(17.2-26.5)\end{array}$ & 0.860 & $\begin{array}{c}90.7 \\
(88.1-93.2)\end{array}$ & 0.872 \\
\hline & Without & $\begin{array}{c}67.4 \\
(64.2-70.6)\end{array}$ & & $\begin{array}{c}54.4 \\
(45.2-63.5)\end{array}$ & & $\begin{array}{c}69.5 \\
(66.1-72.9)\end{array}$ & & $\begin{array}{c}22.5 \\
(17.5-27.4)\end{array}$ & & $\begin{array}{c}90.4 \\
(87.9-92.9)\end{array}$ & \\
\hline \multirow{2}{*}{2} & With & $\begin{array}{c}63.1 \\
(59.8-66.4)\end{array}$ & 0.837 & $\begin{array}{c}60.5 \\
(51.6-69.5)\end{array}$ & 0.892 & $\begin{array}{c}63.5 \\
(60.0-67.1)\end{array}$ & 0.781 & $\begin{array}{c}21.2 \\
(16.8-25.7)\end{array}$ & 0.979 & $\begin{array}{c}90.8 \\
(88.3-93.4)\end{array}$ & 0.961 \\
\hline & Without & $\begin{array}{c}63.6 \\
(60.3-66.9)\end{array}$ & & $\begin{array}{c}59.6 \\
(50.6-68.7)\end{array}$ & & $\begin{array}{c}64.2 \\
(60.7-67.8)\end{array}$ & & $\begin{array}{c}21.3 \\
(16.8-25.8)\end{array}$ & & $\begin{array}{c}90.7 \\
(88.2-93.3)\end{array}$ & \\
\hline
\end{tabular}

PPV = Positive Predictive Value; $\mathbf{N P V}=$ Negative Predictive Value. 
Table 3 - Diagnostic measures according to the Likert categories on mpMRI, for both radiologists, for exams read with and without the dynamic post-contrast sequences.

\begin{tabular}{|c|c|c|c|c|}
\hline Exam & Reader & Category + & Sensitivity & Specificity \\
\hline \multirow{8}{*}{ With contrast } & \multirow{4}{*}{1} & 2 & 100.0 & $1.4(0.5-2.3)$ \\
\hline & & 3 & $57.9(48.8-67.0)$ & $66.4(62.9-69.9)$ \\
\hline & & 4 & $26.3(18.2-34.4)$ & $96.4(95.1-97.8)$ \\
\hline & & 5 & $14.0(7.7-20.4)$ & $99.6(99.1-100.0)$ \\
\hline & \multirow{4}{*}{2} & 2 & 100.0 & $1.3(0.4-2.1)$ \\
\hline & & 3 & $60.5(51.6-69.5)$ & $63.5(60.0-67.1)$ \\
\hline & & 4 & $20.2(12.8-27.5)$ & $95.2(93.6-96.7)$ \\
\hline & & 5 & $13.2(7.0-19.4)$ & $99.4(98.9-100.0)$ \\
\hline \multirow{8}{*}{ Without contrast } & \multirow{4}{*}{1} & 2 & 100.0 & $1.4(0.5-2.3)$ \\
\hline & & 3 & $54.4(45.2-63.5)$ & $69.5(66.1-72.9)$ \\
\hline & & 4 & $27.2(19.0-35.4)$ & $97.9(96.8-98.9)$ \\
\hline & & 5 & $14.0(7.7-20.4)$ & $99.7(99.3-100.0)$ \\
\hline & \multirow{4}{*}{2} & 2 & $97.4(94.4-100.0)$ & $1.3(0.4-2.1)$ \\
\hline & & 3 & $59.6(50.6-68.7)$ & $64.2(60.7-67.8)$ \\
\hline & & 4 & $22.8(15.1-30.5)$ & $94.9(93.2-96.5)$ \\
\hline & & 5 & $13.2(7.0-19.4)$ & 99.3 (98.7-99.9) \\
\hline
\end{tabular}

the exam without post - contrast sequences (4 / $114=3.5 \%)$ for reader 1 and one region more (1 / $114=0.9 \%$ ) for reader 2 . On the other hand, the change of the classification for mpMRI positive (categories 3 to 5) with post - contrast sequences had negative results on biopsy in 22 regions $(22$ / $670=3.3 \%$ ) for reader 1 and in five regions ( 5 | $670=0.7 \%$ ) for reader 2 (Table-1).

A total of 1632 prostatic regions were evaluated by each readers, and the inter - reader agreement was 0.59 (CI: 0.55 - 0.64), demonstrating good agreement. The inter - reader agreement in the per - patient analysis (a total of 102 patients in each exam phase for each reader) was 0.47 (CI: $0.31-0.64)$ on exams without post - contrast sequences and 0.54 (CI: $0.38-0.70$ ) on exams with post - contrast sequences, demonstrating moderate agreement.

\section{DISCUSSION}

Due to over - diagnosis and overtreatment of PCa in the PSA era, mpMRI became useful to detect and characterize prostatic lesions in patients with clinical suspicion for cancer prior to biopsy $(7-10,22)$. The use of MRI to detect CS PCa is already established by many studies performed with complete protocol of mpMRI, including contrast (23-25). Recent studies performed with a complete mpMRI protocol have demonstrated the benefits of MRI over some biomarkers for the detection of $\mathrm{PCa}(10)$ and to monitor candidates for active surveillance $(26,27)$.

As a non - invasive method used for prostatic tumor detection, ideally mpMRI should be as faster and cheaper as possible. It is known that contrast - enhanced mpMRI is more expensive, 
time - consuming, and increases the risk of potential allergic reactions, nephrogenic systemic fibrosis, and gadolinium brain tissue deposition (17-19).

In this prospective study we found similar detection rates for CS PCa in exams read with and without contrast - enhanced sequences with no statistical differences for the five levels of suspicion on mpMRI.

Two recent studies showed high accuracy of MRI for the detection of CS PCa, using a Likert scale with only T2W images and DWI (biparametric - MRI) and PSA levels $(28,29)$. These studies were retrospective, did not categorize the mpMRI suspicion level, and did not compare the results of biparametric - MRI with the gold standard of mpMRI (that includes post - contrast images). In our cohort we included all patients with clinical suspicion of PCa and all mpMRI exams regardless the suspicion level, which probably explains the higher specificity and NPV and lower sensitivity and PPV of our study when compared to their results.

Vargas et al., aiming to evaluate the recommendations in the PI - RADS version 2 and investigate the impact of pathologic tumor volume on PCa detectability on mpMRI, found limited added value of DCE to T2W and DWI sequences (30). Also, few studies showed similar performance for mpMRI with and without contrast media for PCa detection, using both Likert $(31,32)$ and PIRADS (33) scales. These findings corroborate ours that non - contrast mpMRI can improve PCa detection in the near future.

On the other hand, in a study that included only PI - RADS categories 3 and 4, Druskin et al. showed higher positivity for CS PCa in lesions category 3 with and without enhancement (upgraded to PI - RADS 4); however, both lower compared to PI - RADS 4 (32). This finding shows that a PI - RADS 3 lesion with positive enhancement (which is upgraded to PI - RADS 4) has lower risk of CS PCa than a PI - RADS 4 lesion, as showed in a prospective analysis performed by Mertan et al. (34).

We used a Likert scale to stratify the suspicion level on mpMRI, where the radiologist provi- ded a score based on overall impression instead of a fixed criterion. The grade of diffusion restriction (low, moderate, and high) was the most important criteria to classify risk of CS PCa. When this study was designed PI - RADS version 2 had not been published (13) and PI - RADS version 1 was not in use at our institution. The Likert criteria was already shown to be more accurate when applied by readers with previous experience (35). Our study showed moderate to good rates of inter - reader agreement, similar values of those demonstrated using the PI - RADS classification (36-38).

Our study shows that the use of contrast in mpMRI does not increase the detection rate of CS PCa, and has similar accuracy, sensitivity, specificity, PPV, and NPV as compared to a non - contrast protocol. In this prospective study, we included all patients with no prior diagnosis of CS PCa. The diagnostic results yielded consistently high NPV to rule - out CS PCa (>90\%), which could help avoid unnecessary biopsies in patients with low suspicion on mpMRI (categories 1 and 2). Accuracy and specificity were slightly better for non - contrast exams for both readers, but without statistical significance.

This study had several limitations. First, since our institution is an open hospital, a high number of patients (345) did not perform biopsy at our institution and were excluded. Second, our population study included all patients without diagnostic of CS PCa (biopsy naïve, with negative previous biopsy and in active surveillance) and we did not perform a subgroup analysis. Also, we did not use the PIRADS classification; however, previous studies showed good performance of mpMRI using a Likert classification. We used biopsy as a reference standard instead of prostatectomy specimen, what could introduce an imaging - pathology correlation bias; however, many studies were published using this same methodology, with consistent results (39-41). We did not separate peripheral zone and transitional zone tumors. Our temporal resolution for DCE sequences was 13 seconds instead of 10 seconds recommended nowadays. Finally, the short time between the readings could introduce an interpretation bias, but such bias would favor the reading of images that in- 
cluded the contrast - enhanced series, which was performed at the end of the reading session.

In conclusion, our study shows similar performances of mpMRI with and without DCE for CS PCa detection. Further studies should be performed to confirm these results and confirm that a limited, faster, and cheaper mpMRI protocol can be used as standard technique.

\section{ACKNOWLEDGEMENT}

We thank Ana Carolina Cintra Nunes Mafra for helping with the statistical analyses.

\section{Ethical Approval}

IRB approved. For this type of study formal consent is not required.

\section{CONFLICT OF INTEREST}

None declared.

\section{REFERENCES}

1. American Cancer Society. Cancer Facts \& Figures 2016. Atlanta: American Cancer Society; 2016. Available at. <https://www.cancer.org/content/dam/cancer-org/ research/cancer-facts-and-statistics/annual-cancer-factsand-figures/2016/cancer-facts-and-figures-2016.pdf> (accessed December 25, 2017).

2. Dall'Era MA, Cooperberg MR, Chan JM, Davies BJ, Albertsen PC, Klotz LH, et al. Active surveillance for earlystage prostate cancer: review of the current literature. Cancer. 2008;112:1650-9.

3. Howlader N, Noone AM, Krapcho M, et al. SEER Cancer Statistics Review, 1975-2013, National Cancer Institute. Bethesda, MD. Available at: <http://seer.cancer.gov/ csr/1975_2013/> (accessed December 25, 2017).

4. Cohn JA, Wang CE, Lakeman JC, Silverstein JC, Brendler $\mathrm{CB}$, Novakovic KR, et al. Primary care physician PSA screening practices before and after the final U.S. Preventive Services Task Force recommendation. Urol Oncol. 2014;32:41.e23-30.

5. Heijnsdijk EA, Wever EM, Auvinen A, Hugosson J, Ciatto $\mathrm{S}$, Nelen V, et al. Quality-of-life effects of prostate-specific antigen screening. N Engl J Med. 2012;367:595-605.
6. George AK, Turkbey B, Valayil SG, Muthigi A, Mertan F, Kongnyuy $\mathrm{M}$, et al. A urologist's perspective on prostate cancer imaging: past, present, and future. Abdom Radiol (NY). 2016;41:805-16.

7. Siddiqui MM, Rais-Bahrami S, Truong H, Stamatakis $L$, Vourganti S, Nix J, et al. Magnetic resonance imaging/ ultrasound-fusion biopsy significantly upgrades prostate cancer versus systematic 12-core transrectal ultrasound biopsy. Eur Urol. 2013;64:713-9.

8. Monni F, Fontanella P, Grasso A, Wiklund P, Ou YC, Randazzo $M$, et al. Magnetic resonance imaging in prostate cancer detection and management: a systematic review. Minerva Urol Nefrol. 2017;69:567-78.

9. Manfredi M, DE Luca S, Fiori C. Multiparametric prostate MRI for prostate cancer diagnosis: is this the beginning of a new era? Minerva Urol Nefrol. 2017;69:628-9.

10. Porpiglia F, Russo F, Manfredi M, Mele F, Fiori C, Bollito $E$, et al. The roles of multiparametric magnetic resonance imaging, PCA3 and prostate health index-which is the best predictor of prostate cancer after a negative biopsy? J Urol. 2014;192:60-6.

11. Weaver JK, Kim EH, Vetter JM, Fowler KJ, Siegel CL, Andriole GL. Presence of Magnetic Resonance Imaging Suspicious Lesion Predicts Gleason 7 or Greater Prostate Cancer in Biopsy-Naive Patients. Urology. 2016;88:119-24.

12. Salami SS, Ben-Levi E, Yaskiv 0, Ryniker L, Turkbey B, Kavoussi LR, et al. In patients with a previous negative prostate biopsy and a suspicious lesion on magnetic resonance imaging, is a 12-core biopsy still necessary in addition to a targeted biopsy? BJU Int. 2015;115:562-70.

13. Vourganti S, Rastinehad A, Yerram N, Nix J, Volkin D, Hoang $A$, et al. Multiparametric magnetic resonance imaging and ultrasound fusion biopsy detect prostate cancer in patients with prior negative transrectal ultrasound biopsies. J Urol. 2012;188:2152-7.

14. Fütterer $J J$, Briganti $A$, De Visschere P, Emberton $M$, Giannarini G, Kirkham A, et al. Can Clinically Significant Prostate Cancer Be Detected with Multiparametric Magnetic Resonance Imaging? A Systematic Review of the Literature. Eur Urol. 2015;68:1045-53.

15. Barentsz JO, Richenberg J, Clements R, Choyke P, Verma $\mathrm{S}$, Villeirs G, et al. ESUR prostate MR guidelines 2012. Eur Radiol. 2012;22:746-57.

16. Weinreb JC, Barentsz JO, Choyke PL, Cornud F, Haider MA, Macura KJ, et al. PI-RADS Prostate Imaging Reporting and Data System: 2015, Version 2. Eur Urol. 2016;69:16-40. 
17. McDonald RJ, McDonald JS, Kallmes DF, Jentoft ME, Murray DL, Thielen KR, et al. Intracranial Gadolinium Deposition after Contrast-enhanced MR Imaging. Radiology. 2015;275:772-82.

18. ACR Committee on Drugs and Contrast Media. ACR manual on contrast media, version 10.1. Reston, VA 2015:1-129.

19. Kuo PH, Kanal E, Abu-Alfa AK, Cowper SE. Gadoliniumbased MR contrast agents and nephrogenic systemic fibrosis. Radiology. 2007;242:647-9.

20. Epstein JI, Walsh PC, Carmichael M, Brendler CB. Pathologic and clinical findings to predict tumor extent of nonpalpable (stage T1c) prostate cancer. JAMA. 1994;271:368-74.

21. Liang KY, Zeger SL. Longitudinal data analysis using generalized linear models. Biometrika 1986;73:13-22.

22. Siddiqui MM, Rais-Bahrami S, Turkbey B, George AK, Rothwax J, Shakir N, et al. Comparison of MR/ultrasound fusion-guided biopsy with ultrasound-guided biopsy for the diagnosis of prostate cancer. JAMA. 2015;313:390-7.

23. Bonekamp D, Jacobs MA, El-Khouli R, Stoianovici $D$, Macura KJ. Advancements in MR imaging of the prostate: from diagnosis to interventions. Radiographics. 2011;31:677-703.

24. Erbin A, Ozdemir H, Binbay M. Re: Oberlin et al. Diagnostic Value of Guided Biopsies: Fusion and Cognitive-registration Magnetic Resonance Imaging Versus Conventional Ultrasound Biopsy of the Prostate. Urology 2016;92:75-9.

25. Bjurlin MA, Meng $X$, Le Nobin J, Wysock JS, Lepor $H$, Rosenkrantz $A B$, et al. Optimization of prostate biopsy: the role of magnetic resonance imaging targeted biopsy in detection, Iocalization and risk assessment. J Urol. 2014;192:648-58.

26. Dianat SS, Carter HB, Pienta KJ, Schaeffer EM, Landis PK, Epstein JI, et al. Magnetic resonance-invisible versus magnetic resonance-visible prostate cancer in active surveillance: a preliminary report on disease outcomes. Urology. 2015;85:147-53.

27. Johnson LM, Rothwax JT, Turkbey B, Rais-Bahrami S, Wood BJ, Figg WD, et al. Multiparametric magnetic resonance imaging of the prostate aids detect lesion progression. J Comput Assist Tomogr. 2014;38:565-7.

28. Fascelli M, Rais-Bahrami S, Sankineni S, Brown AM, George AK, Ho R, et al. Combined Biparametric Prostate Magnetic Resonance Imaging and Prostate-specific Antigen in the Detection of Prostate Cancer: A Validation Study in a Biopsy-naive Patient Population. Urology. 2016;88:125-34.
29. Rais-Bahrami S, Siddiqui MM, Vourganti S, Turkbey B, Rastinehad AR, Stamatakis $L$, et al. Diagnostic value of biparametric magnetic resonance imaging (MRI) as an adjunct to prostate-specific antigen (PSA)-based detection of prostate cancer in men without prior biopsies. BJU Int. 2015;115:381-8.

30. Vargas HA, Hötker AM, Goldman DA, Moskowitz CS, Gondo T, Matsumoto $\mathrm{K}$, et al. Updated prostate imaging reporting and data system (PIRADS v2) recommendations for the detection of clinically significant prostate cancer using multiparametric MRI: critical evaluation using whole-mount pathology as standard of reference. Eur Radiol. 2016;26:1606-12.

31. Barth BK, De Visschere PJL, Cornelius A, Nicolau C, Vargas HA, Eberli D, et al. Detection of Clinically Significant Prostate Cancer: Short Dual-Pulse Sequence versus Standard Multiparametric MR Imaging-A Multireader Study. Radiology. 2017;284:725-36.

32. Mussi TC, Martins T, Garcia RG, Filippi RZ, Lemos GC, Baroni RH. Are Dynamic Contrast-Enhanced Images Necessary for Prostate Cancer Detection on Multiparametric Magnetic Resonance Imaging? Clin Genitourin Cancer. 2017;15:e447-e54.

33. Kuhl CK, Bruhn R, Krämer N, Nebelung S, Heidenreich A Schrading S. Abbreviated Biparametric Prostate MR Imaging in Men with Elevated Prostate-specific Antigen. Radiology. 2017;285:493-505.

34. Mertan FV, Greer MD, Shih JH, George AK, Kongnyuy M, Muthigi A, et al. Prospective Evaluation of the Prostate Imaging Reporting and Data System Version 2 for Prostate Cancer Detection. J Urol. 2016;196:690-6.

35. Rosenkrantz AB, Lim RP, Haghighi M, Somberg MB, Babb JS, Taneja SS. Comparison of interreader reproducibility of the prostate imaging reporting and data system and likert scales for evaluation of multiparametric prostate MRI. AJR Am J Roentgenol. 2013;201:W612-8.

36. Zhao C, Gao G, Fang D, Li F, Yang X, Wang H, et al. The efficiency of multiparametric magnetic resonance imaging (mpMRI) using PI-RADS Version 2 in the diagnosis of clinically significant prostate cancer. Clin Imaging. 2016;40:885-8.

37. Rosenkrantz AB, Ginocchio LA, Cornfeld D, Froemming AT, Gupta RT, Turkbey B, et al. Interobserver Reproducibility of the PIRADS Version 2 Lexicon: A Multicenter Study of Six Experienced Prostate Radiologists. Radiology. 2016;280:793-804.

38. Muller BG, Shih JH, Sankineni S, Marko J, Rais-Bahrami S, George AK, et al. Prostate Cancer: Interobserver Agreement and Accuracy with the Revised Prostate Imaging Reporting and Data System at Multiparametric MR Imaging. Radiology. 2015;277:741-50 
39. Rosenkrantz AB, Verma S, Choyke P, Eberhardt SC, Eggener $\mathrm{SE}$, Gaitonde K, et al. Prostate Magnetic Resonance Imaging and Magnetic Resonance Imaging Targeted Biopsy in Patients with a Prior Negative Biopsy: A Consensus Statement by AUA and SAR. J Urol. 2016;196:1613-8.

40. Rosenkrantz AB, Babb JS, Taneja SS, Ream JM. Proposed Adjustments to PI-RADS Version 2 Decision Rules: Impact on Prostate Cancer Detection. Radiology. 2017;283:119-29.
41. Nam RK, Wallis CJ, Stojcic-Bendavid J, Milot L, Sherman C, Sugar L, et al. A Pilot Study to Evaluate the Role of Magnetic Resonance Imaging for Prostate Cancer Screening in the General Population. J Urol. 2016;196:361-6.

\section{Correspondence address:}

Thais Caldara Mussi, MD

Departamento de Radiologia e Diagnóstico por Imagem

Hospital Israelita Albert Einstein

Av. Albert Einstein, n ${ }^{\circ} 627$

São Paulo, SP, 05652-900, Brasil

Telephone: + 5511 2151-4271

E-mail: thaiscaldara@gmail.com 